\title{
Fuzzy Multi Criteria Decision For Industriel Zone Selection
}

\author{
Boudaoud Lakhdar El Amine \\ Computer science Laboratory of Oran (LIO), University of Oran1 Ahmed Ben Bella \\ blakhdarelamine@gmail.com
}

\begin{abstract}
Fuzzy Multi Criteria Decision is process of Selection and range, However they have different way to use it in Decisional Problem, our situation is to Select the best Zone Between the others looking the Criteria of Temperature, Socio Economic, Acclimatization, Population, to select the adequate Alternative by using The AHP Method proposed by Saaty [3], also, we have added the FAHP (Fuzzy AHP) Method, it's for more precision and exactly result.
\end{abstract}

Keyword: Fuzzy Logic, AHP, Fuzzy Multi Criteria Decision.

\section{INTRODUCTION}

Multi Criteria Decision is one of rich domain developed for the decider, Environment Criteria , Climate, Socio Economic Criteria, and Temperature are the selected factors for the installation site in Algeria, AHP (Analytical Hierarchy Process) is the crucial tools for the Analytical Process and the Ranking of this Alternatives with Saaty Echelle [3] , also this problem is with height complexity of four Criteria and five Alternative.

Fuzzy Multi Criteria Decision is appliqued on the same problems with choosing of Representation Model between tow way, Rectangular Membership Model [3] or Trapezoidal Membership Model.

This Paper is object to provide the final resolve of Alternative ranking with tow Method, the AHP Solution, it's based on The Select Weight of each Criteria and Alternative [3], then, the FAHP [5] Second solution to take the best decision with The selection of each Fuzzy Criteria and Fuzzy Alternative.

The combine Between Fuzzy Logic and AHP is to studies more precision of the choice of site and Found the Site Ranking.
The remainder of this article is presented as Follow: Section 2 is focused to the Related Work who can be devised on Theory Research and applied Research, also, The section 3 explain the proposal approach and Our Fuzzy Decision Support System (FDSS), The Conception is represented in section 4 , we concluded by Conclusion and Perspectives.

\section{RELATED WORK}

Working on Multi Criteria Decision may be have certain number of thing :

\section{State of art and Theory}

The work in AHP and FAHP Context is remounted on 2008 with M.H. Vahidnia, A. Alesheikh, A. Alimohammadi, and A. Bassiri [2], they have included the Spatial Criteria of Hydrology and water resources, Forestry, Transportation, Agriculture, Natural hazard management, Health care resource allocation and T.Aissa, A. Baghdad in 2017, have Introduce four Spatial Criteria ( Economic Criteria, Temperature, Climate and Environment Criteria), and about the Alternative they have use local Actions.

\section{Development state}

The work of 2008 has concentered on the FAHP implementation based on theory AHP, never model have used in this time (2008) but in [6], They have worked with the Triangular Function , The MCDA method have resulted more than one result and they have displayed three result for each one Local Alternatives .

About the mixed integration between the AHP and GIS , they have visualized the spatial Alternative and the Spatial Criteria in two way, in the input and output there are displaying of Site Alternatives 


\section{Pairwise comparison}

\section{BACKGROUND}

\section{MCDM and GIS}

GIS and MCDM are currently the two most common decision support tools employed to solve spatial decision-making problems. GIS is a computer-based technology and methodology for collecting, managing, analyzing, modeling, and presenting geographic data for a wide range of applications [3]. MCDM techniques are decision support tools designed to analyze decision problems, generate useful alternative solutions, and evaluate alternatives based on the decision maker's values and preferences. The general objective of these methods is to assist the decision maker in selecting the best alternative from the number of feasible alternatives under the presence of multiple choice criteria and diverse criteria priorities [8, 15]. A literature review suggests that a number of approaches have been used in formulating MCDM tools.

In this Research, we have used AHP like subjective Method who can set decision in the restricted environments, with the GIS it was one of the procedure MCDM

\section{AHP}

Analytic Hierarchy Process (AHP) is one of Multi Criteria decision making method that was originally developed by Prof. Thomas L. Saaty. In short, it is a method to derive ratio scales from paired comparisons. The input can be obtained from actual measurement such as price, weight etc., or from subjective opinion such as satisfaction feelings and preference. AHP allow some small inconsistency in judgment because human is not always consistent. The ratio scales are derived from the principal Eigen vectors and the consistency index is derived from the principal Eigen value.
For each element of the hierarchy structure all the associated elements in low hierarchy are compared in to attributes comparison matrices as follows:

$$
A=\left(\begin{array}{cccc}
1 & \frac{w_{1}}{w_{2}} & \frac{w_{1}}{w_{j}} & \frac{w_{1}}{w_{n}} \\
\frac{w_{2}}{w_{1}} & \frac{1}{1} & \frac{w_{2}}{w_{j}} & \frac{w_{2}}{w_{n}} \\
\frac{w_{j}}{w_{1}} & \frac{w_{j}}{w_{2}} & \frac{1}{1} & \frac{w_{i}}{w_{n}} \\
\frac{w_{n}}{w_{1}} & \frac{w_{n}}{w_{2}} & \frac{w_{n}}{w_{i}} & \frac{1}{1}
\end{array}\right)
$$

Where $A=$ comparison pairwise matrix, $\mathrm{w}_{1}=$ wheight of element 1 , $\mathrm{w}_{2}=$ wheight of element 2 , $\mathrm{w}_{\mathrm{i}}=$ wheight of element $\mathrm{I}$, $w_{j}=$ wheight of element $j$, $w_{n}=$ wheight of element $n$.

in order to determine the relative preferences for two elements of the hierarchy in matrix A, an underlying semantical scale is employs with values from 1 to 9 to rate (Table 1).

\begin{tabular}{|l|l|}
\hline $\begin{array}{l}\text { Preferences expressed in } \\
\text { numeric variables }\end{array}$ & $\begin{array}{l}\text { Preferences expressed } \\
\text { in linguistic variables }\end{array}$ \\
\hline 1 & Equal importance \\
\hline 3 & Moderate importance \\
\hline 5 & Strong importance \\
\hline 7 & Very strong importance \\
\hline 9 & Extreme importance \\
\hline $2,4,6,8$ & $\begin{array}{l}\text { Intermediate values between } \\
\text { adjacent scale values }\end{array}$ \\
\hline
\end{tabular}

\subsection{Estimate the relative weights}

Some methods like eigenvalue method are used to calculate the relative weights of elements in each pairwise comparison matrix. The relative weights $(W)$ of matrix $A$ is obtained from following equation:

$$
\left(A-\lambda_{\max } I\right) X W=0
$$

Where $\lambda \max =$ the biggest eigenvalue of matrix $\mathrm{A}$,

$I=$ unit matrix 


\subsection{Check the consistency}

In this step the consistency property of matrices is checked to ensure that the judgments of decision makers are consistent. For this end some pre-parameter is needed. Consistency Index $(C I)$ is calculated as:

$C I=\frac{\lambda_{\max }-n}{n-1}$

The consistency index of a randomly generated reciprocal matrix shall be called to the random index $(R I)$, with reciprocals forced. An average $R I$ for the matrices of order 1-15 was generated by using a sample size of 100 [18]. The table of random indexes of the matrices of order 1-15 can be seen in Saaty (1980). The last ratio that has to be calculated is $C R$ (Consistency Ratio). Generally, if $C R$ is less than 0.1, the judgments are consistent, so the derived weights can be used. The formulation of

$C R$ is:

$$
C R=\frac{C I}{R I}
$$

\subsection{Obtain the overall rating}

In last step the relative weights of decision elements are aggregated to obtain an overall rating for the alternatives as follow:

$W_{i}^{S}=\sum_{j=1}^{j=m} W_{i j}^{S} w_{j}^{a}$

Where $W_{i}^{s}$ is total weight of site $i$

$W_{i j}^{S}$ : weight of Alternatives (site) $i$

Associated to Attribute $\mathrm{j}$ (map layer)

$w_{j}^{a}$ : Weight of attribute $j$

$\mathrm{m}$ :number of attribute

$\mathrm{n}$ : number of site

\section{FAHP}

$\delta(t)$

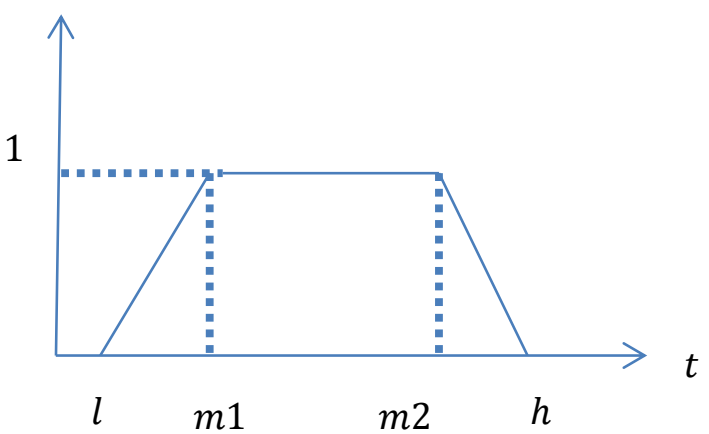

3.2.1 Fuzzy Trapézoïdal Function

$$
\delta(t)=\left\{\begin{array}{cl}
0 \quad s i \quad 0<t<l \quad \text { or } h<t \\
\frac{m 1-l}{t-l} & \text { si } l<t<m 1 \\
1 \quad s i & m 1<t<m 2 \\
\frac{h-m 2}{t-h} & \text { si } m 2<t<h
\end{array}\right.
$$

There are more Fuzzy operation applied in this domain, we considered two Operand A and B;

$A=(a, b, c, d)$

$B=(e, f, g, p)$ Then

$A \boxplus B=(a+e, b+f, c+g, d+p)$

$A \boxminus B=(a-e, b-f, c-g, d-p)$

$A \bigotimes B=(a * e, b * f, c * g, d * p)$

$A \oslash B=(a / e, b / f, c / g, d / p)$

Let considered $\delta^{i}(x 1)=\sum_{i=1}^{n} \delta_{i}(x)$ Then :

$\delta_{1}=\operatorname{TRAP}(l 1, m 1, m 2, h 1)$;

$\delta_{2}=\operatorname{TRAP}(l 2, m 3, m 4, h 2)$,

here Representation is below :

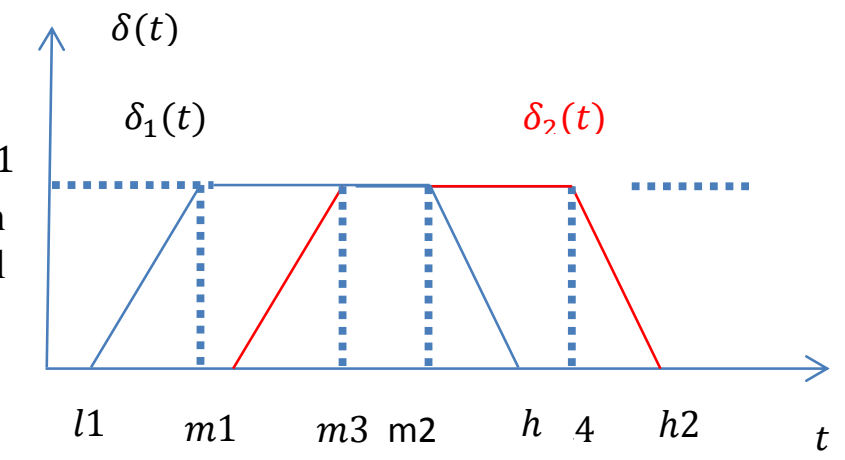

$\sum_{i=1}^{n} \sum_{j=1}^{m} \delta_{g_{i}}^{j}$

$=\sum_{i=1}^{n}\left(\sum_{i=1}^{n} l_{i}, \sum_{i=1}^{n} m_{2 * i+1}, \sum_{i=1}^{n} m_{2 * i}, \sum_{i=1}^{n} h_{i}\right)$

$$
\begin{aligned}
& \left(\sum_{i=1}^{n} \sum_{j=1}^{m} \delta_{g_{i}}^{j}\right)^{-1}= \\
& \left(\frac{1}{\sum_{i=1}^{n} h_{i}}, \frac{1}{\sum_{i=1}^{n} m_{2 * i}}, \frac{1}{\sum_{i=1}^{n} m_{2 * i+1}}, \frac{1}{\sum_{i=1}^{n} l_{i}}\right)
\end{aligned}
$$

\section{PROPOSED APPROACH}

In this section, we present the main aspect of our contribution. As mentioned in the previous section, Salem Chakhar [1] in proposes three integration modes, indirect integration, built-in integration and full integration. In this paper a mixed integration is proposed: Preparing geographic criteria to support decision 
making in weighting is made by GIS beginning of the decision-making process, independently (indirect integration) in zones studies begins by collecting data about screening phase while visualization criteria and actions from geographic, function is integrated directly (full socioeconomic and climate databases as integration) in the MCDA module in the archives of regions, the maps for the criteria evaluation phase and is considered as a finality of the decision analysis.

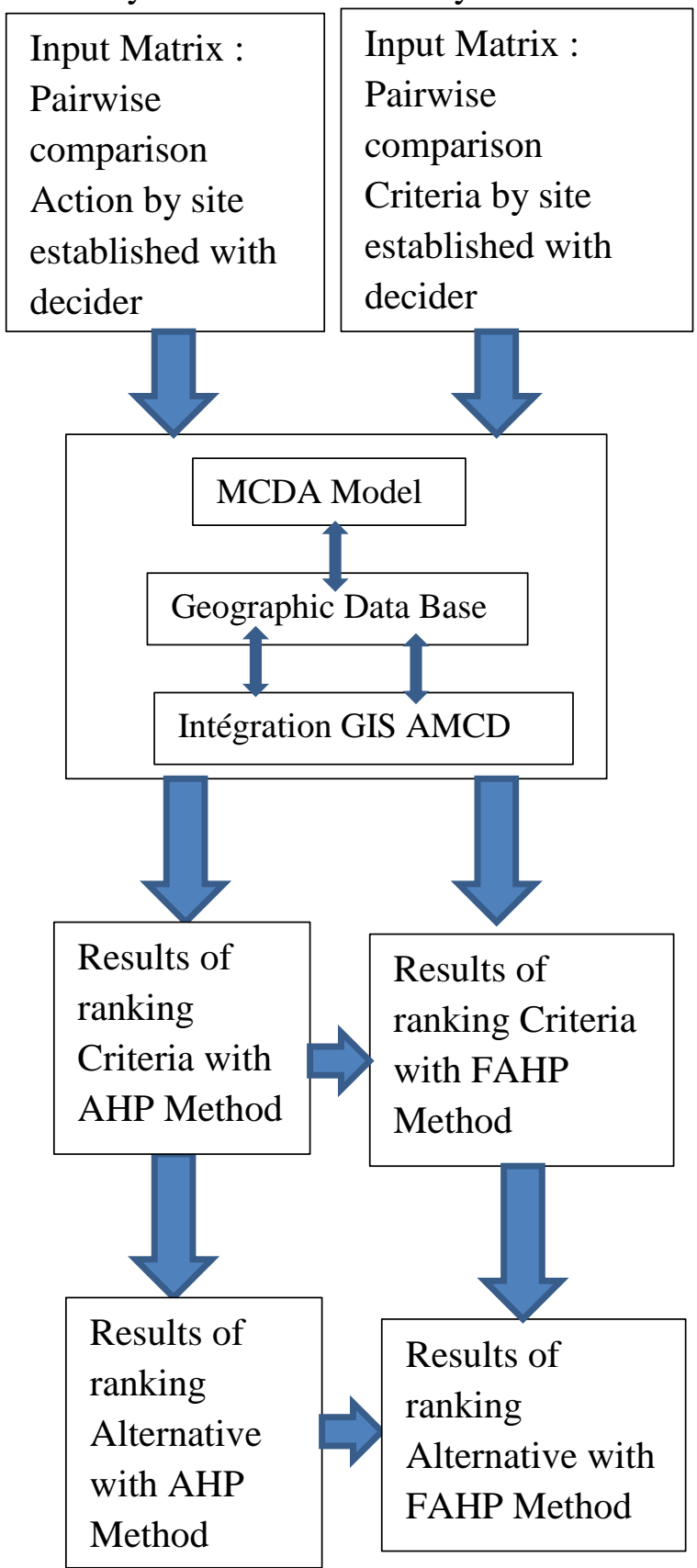

Figure 4 : Our Model MCDA

The proposed approach consists of two phases: Screening and evaluation as explained below and depicted in (Fig. 3)

- Screening phase: After the choice of Criteria number level one, Criteria number level two, Criteria number three, Action Number, at the are built using GIS. A field of expertise is available. Then with Our Interface , we can displaying the problem representing as tree * The AHP procedure involves six essential steps $[2,3]$ :

1. Definition of the problem

2. Represent the problem by an hierarchy structure

3. Construct pairwise comparison matrixes

4. Estimate the relative weights

5. Check the consistance

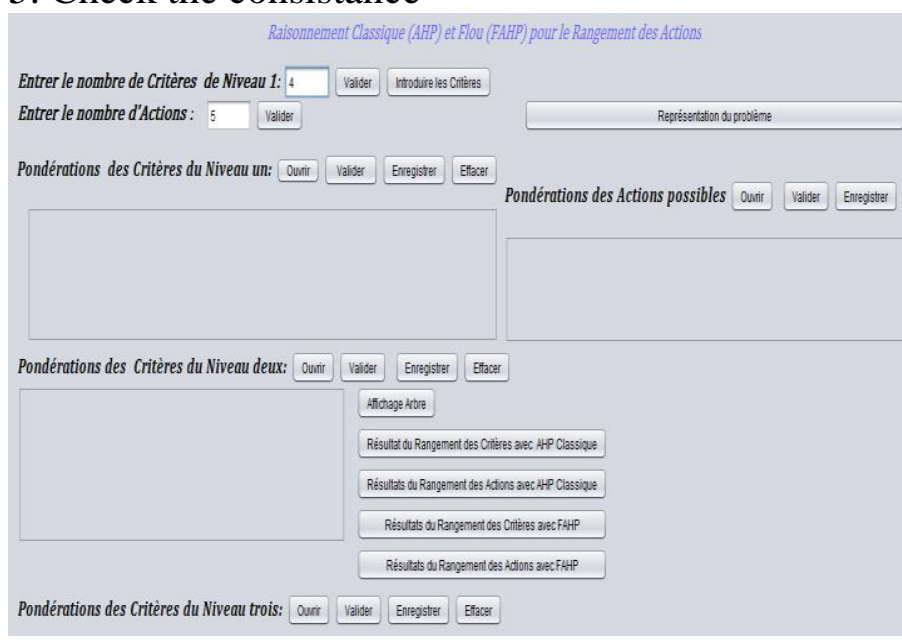

\section{Figure 03 : Introduction Input}

After input data, we will displaying :

1. Tree representation

2. Ranking Criteria level one with AHP Method

3. Ranking Criteria level two with AHP Method

4. Ranking Action with AHP Method

5. Ranking Criteria level one with FAHP Method

6. Ranking Criteria level two with FAHP Method

7. Ranking Action with FAHP Method

8. Visualization site with mapping

\section{Tree representation}




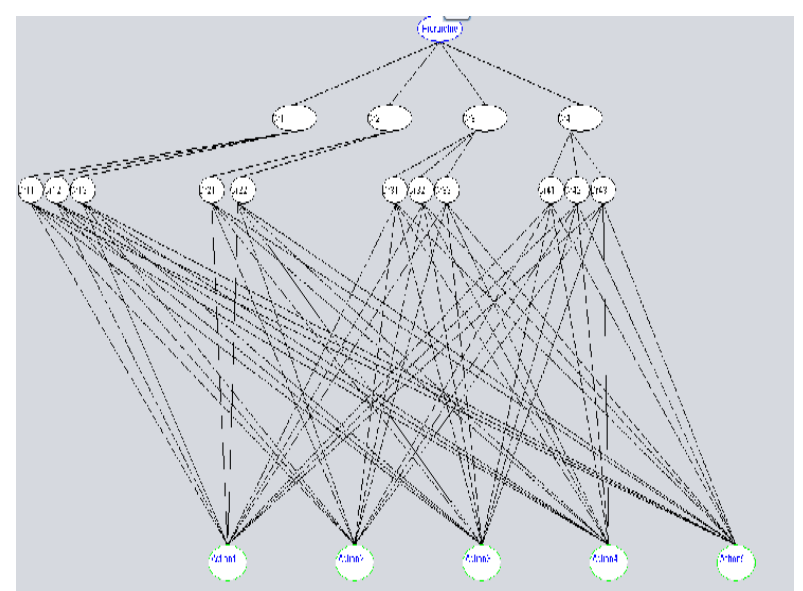

Figure 05 : Tree Decision representation

In this hierarchy there are Four (4) Criteria in level one and in the First Criteria there are three Criteria of level two, In the Second Criteria there are two Criteria level two, in the third criteria of level one, there are three Criteria of level two, Finally the fourth criteria have three criteria of level two, then this hierarchy has four actions.

Then we can say that the First Criteria is environmental impact Criteria, the second Criteria is represented by the natural hazard Criteria, The third Criteria is climate, Finally the fourth criteria is Socio economy

We have study her variability with Normalization, also firstly I have posed SAATY ponderation for the fourth Criteria like below:

1. Saaty Pairwise of Criteria level one in the Naama City

\begin{tabular}{|l|l|l|l|l|l|l|}
\hline CT1 & IE & NH & C & S & $\%$ & Rang \\
\hline IE & 1 & $1 / 7$ & 3 & $1 / 3$ & 0.22 & 4 \\
\hline NH & 7 & 1 & $1 / 7$ & $1 / 2$ & 2.16 & 3 \\
\hline C & $1 / 3$ & 7 & 1 & $1 / 3$ & 2.16 & 2 \\
\hline S & 3 & 2 & 3 & 1 & 2.25 & 1 \\
\hline
\end{tabular}

IE : environmental impact Criteria

$\mathrm{NH}$ : natural hazard Criteria

$\mathrm{C}$ : Climate Criteria

S: Socio Economic Criteria

2. Saaty Pairwise of Criteria level one in the Horchaia City

\begin{tabular}{|l|l|l|l|l|l|l|}
\hline $\mathrm{CT} 2$ & $\mathrm{IE}$ & $\mathrm{NH}$ & $\mathrm{C}$ & $\mathrm{S}$ & $\%$ & $\mathrm{Rg}$ \\
\hline $\mathrm{IE}$ & 1 & $1 / 2$ & 4 & $1 / 3$ & 1.4 & 3 \\
\hline $\mathrm{NH}$ & 2 & 1 & 7 & $1 / 3$ & 2.5 & 1 \\
\hline $\mathrm{C}$ & $1 / 4$ & $1 / 7$ & 1 & $1 / 2$ & 0.4 & 4 \\
\hline $\mathrm{S}$ & 3 & 3 & 2 & 1 & 2.2 & 2 \\
\hline
\end{tabular}

3. Saaty Pairwise of Criteria level one in Ras ElMa City

\begin{tabular}{|l|l|l|l|l|l|l|}
\hline CT3 & IE & NH & C & S & $\%$ & $\mathrm{Rg}$ \\
\hline IE & 1 & $1 / 3$ & 1 & $1 / 2$ & 0.7 & 4 \\
\hline NH & 3 & 1 & $1 / 9$ & $1 / 3$ & 1.1 & 3 \\
\hline $\mathrm{C}$ & 1 & 9 & 1 & $1 / 4$ & 2.8 & 1 \\
\hline S & 2 & 3 & 4 & 1 & 2.5 & 2 \\
\hline
\end{tabular}

4. Saaty Pairwise of Criteria level one in Maghnia City

\begin{tabular}{|l|l|l|l|l|l|l|}
\hline $\mathrm{CT} 4$ & $\mathrm{IE}$ & $\mathrm{NH}$ & $\mathrm{C}$ & $\mathrm{S}$ & $\%$ & $\mathrm{Rg}$ \\
\hline $\mathrm{IE}$ & 1 & $1 / 9$ & 2 & $1 / 4$ & 0.8 & 4 \\
\hline $\mathrm{NH}$ & 9 & 1 & $1 / 5$ & $1 / 2$ & 2.6 & 1 \\
\hline $\mathrm{C}$ & $1 / 2$ & 5 & 1 & 1 & 1.8 & 3 \\
\hline $\mathrm{S}$ & 4 & 3 & 1 & 1 & 2.2 & 2 \\
\hline
\end{tabular}

This Ponderation has been injected by the decider, In the second level, we found three Sub-Criteria $(\mathrm{C} 1, \mathrm{C} 2, \mathrm{C} 3)$ in IE, two Sub-Criteria $(\mathrm{C} 4, \mathrm{C} 5)$ in $\mathrm{NH}$, Three Sub-Criteria $(\mathrm{C} 6, \mathrm{C} 7, \mathrm{C} 8)$ in $\mathrm{C}$, Finally Three Sub Criteria $(\mathrm{C} 9, \mathrm{C} 10, \mathrm{C} 11)$ in $\mathrm{S}$.

Now, The decider is invited to resolve The Sub-Criteria Ponderation

Ponderation of Sub-Criteria in IE

\begin{tabular}{|l|l|l|l|l|l|}
\hline IE & C1 & C2 & C3 & $\%$ & $\mathrm{Rg}$ \\
\hline C1 & 1 & 5 & 5 & 3.6 & 1 \\
\hline C2 & $1 / 5$ & 1 & 1 & 0.7 & 2 \\
\hline C3 & $1 / 5$ & 1 & 1 & 0.7 & 2 \\
\hline
\end{tabular}

C1 : Pollution risk water table

C2 : Fauna and Flora

C3 : Citizen noise pollution

Pairwise of Sub-Criteria in NH

\begin{tabular}{|l|l|l|l|l|}
\hline NH & C4 & C5 & $\%$ & Rg \\
\hline C4 & 1 & 5 & 3 & 1 \\
\hline C5 & $1 / 5$ & 1 & 0.56 & 2 \\
\hline
\end{tabular}

C4: Seismicity

C5 : Flood

Pairwise of sub-Criteria in $\mathbf{C}$

\begin{tabular}{|l|l|l|l|l|l|}
\hline $\mathrm{C}$ & $\mathrm{C} 6$ & $\mathrm{C} 7$ & $\mathrm{C} 8$ & $\%$ & $\mathrm{Rg}$ \\
\hline C6 & 1 & 3 & 3 & 2.33 & 1 \\
\hline C7 & $1 / 3$ & 1 & 3 & 1.44 & 2 \\
\hline C8 & $1 / 3$ & $1 / 3$ & 1 & 0.5 & 3 \\
\hline
\end{tabular}

C6 : Temperature

C7 : pluviometry

C8 : Bioclimatic floor 
Pairwise of Action in C6

Pairwise of sub-Criteria in $\mathbf{S}$

\begin{tabular}{|l|l|l|l|l|l|}
\hline S & C9 & C10 & C11 & $\%$ & $\mathrm{Rg}$ \\
\hline C9 & 1 & $1 / 3$ & $1 / 5$ & 0.48 & 3 \\
\hline C10 & 3 & 1 & 3 & 2.33 & 1 \\
\hline C11 & 5 & $1 / 3$ & 1 & 2.11 & 2 \\
\hline
\end{tabular}

C9 : Development cost

C10 : Development potential

C11 : Transport infrastructure

Then, the decider pandurate the Action as below :

Pairwise Of Action in C1 :

\begin{tabular}{|l|l|l|l|l|l|l|}
\hline C1 & CT1 & CT2 & CT3 & CT4 & CT5 & Rg \\
\hline CT1 & 1 & 1 & 1 & $1 / 2$ & 2 & 1.1 \\
\hline CT2 & 1 & 1 & 1 & $1 / 3$ & 1 & 0.86 \\
\hline CT3 & 1 & 1 & 1 & $1 / 2$ & 1 & 0.9 \\
\hline CT4 & 2 & 3 & 2 & 1 & 2 & 2 \\
\hline CT5 & $1 / 2$ & 1 & 1 & $1 / 2$ & 1 & 0.8 \\
\hline
\end{tabular}

Pairwise Of Action in C2 :

\begin{tabular}{|l|l|l|l|l|l|l|}
\hline C2 & CT1 & CT2 & CT3 & CT4 & CT5 & Rg \\
\hline CT1 & 1 & $1 / 5$ & $1 / 3$ & $1 / 3$ & $1 / 4$ & 0.4 \\
\hline CT2 & 5 & 1 & $1 / 2$ & 1 & $1 / 2$ & 1.6 \\
\hline CT3 & 3 & 2 & 1 & 1 & $1 / 2$ & 1.5 \\
\hline CT4 & 3 & 1 & 1 & 1 & $1 / 2$ & 1.3 \\
\hline CT5 & 4 & 2 & 2 & 2 & 1 & 2.2 \\
\hline
\end{tabular}

Pairwise Of Action in C3

\begin{tabular}{|l|l|l|l|l|l|l|}
\hline C3 & CT1 & CT2 & CT3 & CT4 & CT5 & Rg \\
\hline CT1 & 1 & 5 & 4 & 3 & 5 & 3.6 \\
\hline CT2 & $1 / 5$ & 1 & $1 / 2$ & $1 / 3$ & $1 / 2$ & 0.49 \\
\hline CT3 & $1 / 4$ & 2 & 1 & $1 / 2$ & 4 & 1.51 \\
\hline CT4 & $1 / 3$ & 3 & 2 & 1 & 5 & 2.26 \\
\hline CT5 & $1 / 5$ & 2 & $1 / 4$ & $1 / 5$ & 1 & 0.69 \\
\hline
\end{tabular}

\begin{tabular}{|l|l|l|l|l|l|l|}
\hline \multicolumn{7}{|c|}{ Pairwise of Action in C4 } \\
\hline C4 & CT1 & CT2 & CT3 & CT4 & CT5 & Rg \\
\hline CT1 & 1 & 1 & 1 & 1 & 3 & 1.4 \\
\hline CT2 & 1 & 1 & 1 & 1 & 3 & 1.75 \\
\hline CT3 & 1 & 1 & 1 & 1 & 3 & 1.75 \\
\hline CT4 & 1 & 1 & 1 & 1 & 3 & 1.75 \\
\hline CT5 & $1 / 3$ & $1 / 3$ & $1 / 3$ & $1 / 3$ & 1 & 0.46 \\
\hline
\end{tabular}

Pairwise of Action in C5

\begin{tabular}{|c|c|c|c|c|c|c|c|c|c|c|c|c|c|}
\hline 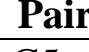 & $9 \mathrm{~m}$ & CTO & 79 & & & & CT5 & $1 / 3$ & $1 / 2$ & $1 / 3$ & $1 / 3$ & 1 & 0.5 \\
\hline C5 & CT1 & CT2 & CT3 & CT4 & CT5 & $\mathrm{Rg}$ & & & & & & & \\
\hline CT1 & 1 & $1 / 2$ & $1 / 3$ & $1 / 4$ & 2 & & \multirow{5}{*}{\multicolumn{7}{|c|}{$\begin{array}{l}\text { Deduction of Alternative AHP Rang } \\
\text { Rang }(C T \mathbf{1})=(((I E * C 1 * C T \mathbf{1})+ \\
(I E * C 2 * C T \mathbf{1})+(I E * C 3 * C T \mathbf{1}))+ \\
((N H * C 4 * C T \mathbf{1})+(N H * C 5 * C T 1))+\end{array}$}} \\
\hline CT2 & 2 & 1 & $1 / 2$ & $1 / 3$ & 1 & 0.96 & & & & & & & \\
\hline CT3 & 3 & 2 & 1 & $1 / 2$ & 2 & 1.7 & & & & & & & \\
\hline CT4 & 4 & 3 & 2 & 1 & 3 & 2.6 & & & & & & & \\
\hline CT5 & $1 / 2$ & 1 & $1 / 2$ & $1 / 3$ & 1 & 0.66 & & & & & & & \\
\hline
\end{tabular}

Pairwise of Action in C7

Pairwise of Action in C8

Pairwise of Action in C9

Pairwise of Action in C10

Pairwise of Action in C11

\begin{tabular}{|l|l|l|l|l|l|l|}
\hline C11 & CT1 & CT2 & CT3 & CT4 & CT5 & Rg \\
\hline CT1 & 1 & 5 & 1 & 1 & 3 & 2.2 \\
\hline CT2 & $1 / 5$ & 1 & $1 / 5$ & $1 / 5$ & 2 & 0.72 \\
\hline CT3 & 1 & 5 & 1 & 1 & 3 & 2.2 \\
\hline CT4 & 1 & 5 & 1 & 1 & 3 & 2.2 \\
\hline CT5 & $1 / 3$ & $1 / 2$ & $1 / 3$ & $1 / 3$ & 1 & 0.5 \\
\hline
\end{tabular}

\begin{tabular}{|l|l|l|l|l|l|l|}
\hline C6 & CT1 & CT2 & CT3 & CT4 & CT5 & Rg \\
\hline CT1 & 1 & 3 & 2 & 1 & 2 & 1.8 \\
\hline CT2 & $1 / 3$ & 1 & 3 & 3 & 5 & 2.46 \\
\hline CT3 & $1 / 2$ & $1 / 3$ & 1 & $1 / 2$ & 1 & 0.66 \\
\hline CT4 & 1 & $1 / 3$ & 2 & 1 & 2 & 1.26 \\
\hline CT5 & $1 / 2$ & $1 / 5$ & 1 & $1 / 2$ & 1 & 0.62 \\
\hline
\end{tabular}

\begin{tabular}{|l|l|l|l|l|l|l|}
\hline C7 & CT1 & CT2 & CT3 & CT4 & CT5 & Rg \\
\hline CT1 & 1 & 3 & $1 / 3$ & $1 / 2$ & 7 & 2.36 \\
\hline CT2 & $1 / 3$ & 1 & $1 / 4$ & $1 / 2$ & 6 & 1.61 \\
\hline CT3 & 3 & 4 & 1 & 2 & 8 & 3.6 \\
\hline CT4 & 2 & 2 & $1 / 2$ & 1 & 6 & 2.3 \\
\hline CT5 & $1 / 7$ & $1 / 6$ & $1 / 8$ & $1 / 6$ & 1 & 0.31 \\
\hline
\end{tabular}

\begin{tabular}{|l|l|l|l|l|l|l|}
\hline C8 & CT1 & CT2 & CT3 & CT4 & CT5 & Rg \\
\hline CT1 & 1 & 1 & 1 & 1 & 3 & 1.4 \\
\hline CT2 & 1 & 1 & 1 & 1 & 3 & 1.4 \\
\hline CT3 & 1 & 1 & 1 & 1 & 3 & 1.4 \\
\hline CT4 & 1 & 1 & 1 & 1 & 3 & 1.4 \\
\hline CT5 & $1 / 3$ & $1 / 3$ & $1 / 3$ & $1 / 3$ & 1 & 0.46 \\
\hline
\end{tabular}

\begin{tabular}{|l|l|l|l|l|l|l|}
\hline C9 & CT1 & CT2 & CT3 & CT4 & CT5 & Rg \\
\hline CT1 & 1 & $1 / 3$ & 2 & $1 / 3$ & $1 / 5$ & 0.75 \\
\hline CT2 & 3 & 1 & 5 & 3 & $1 / 3$ & 2.46 \\
\hline CT3 & $1 / 2$ & $1 / 5$ & 1 & $1 / 2$ & $1 / 5$ & 0.44 \\
\hline CT4 & 3 & 3 & $1 / 3$ & 1 & 6 & 2.66 \\
\hline CT5 & 5 & 3 & 5 & $1 / 6$ & 1 & 2.82 \\
\hline
\end{tabular}

\begin{tabular}{|l|l|l|l|l|l|l|}
\hline C10 & CT1 & CT2 & CT3 & CT4 & CT5 & Rg \\
\hline CT1 & 1 & 5 & 1 & 2 & 3 & 2.4 \\
\hline CT2 & $1 / 5$ & 1 & $1 / 5$ & 3 & 2 & 1.28 \\
\hline CT3 & 1 & 5 & 1 & $1 / 3$ & 3 & 2.06 \\
\hline CT4 & $1 / 2$ & $1 / 3$ & 3 & 1 & 4 & 1.76 \\
\hline CT5 & $1 / 3$ & $1 / 2$ & $1 / 3$ & $1 / 4$ & 1 & 0.48 \\
\hline
\end{tabular}


$((C * C 6 * C T 1)+(C * C 7 * C T 1)+$

$(C * C 8 * C T 1)+(S * C 9 * C T 1)+$

$((0.22 * 3.6 * 1.1)+(1.4 * 0.7 * 0.4)+$

$(0.7 * 0.7 * 0.6)+(2.16 * 3 * 1.4)+$

$(2.5 * 0.56 * 0.81)+(2.16 * 2.33 *$

$1.8)+(0.4 * 1.44 * 3.36)+(2.8 * 0.5 *$

$1.4)+(2.25 * 0.48 * 0.75)+$

$(2.2 * 2.33 * 2.4)+(2.5 * 2.11 * 2.2))=$

$49.43 / 220=0.21$

$\operatorname{Rang}(C T 2)=(((I E * C 1 * C T 2)+$

$(I E * C 2 * C T 2)+(I E * C 3 * C T 2))+$

$((\mathrm{NH} * \mathrm{C4} * \mathrm{CT} 2)+(\mathrm{NH} * \mathrm{C5} * \mathrm{CT} 2))+$

$((C * C 6 * C T 2)+(C * C 7 * C T 2)+$

$(C * C 8 * C T 2)+(S * C 9 * C T 2)+$

$(S * C 10 * C T 2)+(S * C 11 * C T 2))=$

$((0.22 * 3.6 * 0.86)+(1.4 * 0.7 * 1.6)+$

$(0.7 * 0.7 * 0.49)+(2.16 * 3 * 1.75)+$

$(2.5 * 0.56 * 0.96)+(2.16 * 2.33 *$

$2.48)+(0.4 * 1.44 * 1.61)+$

$(2.8 * 0.5 * 1.4)+(2.25 * 0.48 * 2.46)+$

$(2.2 * 2.33 * 1.28)+(2.5 * 2.11 *$

$0.72))=(43.56) / 220=0.2$

$\operatorname{Rang}(C T 3)=(((I E * C 1 * C T 3)+$

$(I E * C 2 * C T 3)+(I E * C 3 * C T 3))+$
$(S * C 10 * C T 1)+(S * C 11 * C T 1))=$

$1.4)+(2.25 * 0.48 * 2.66)+$

$(2.2 * 2.33 * 1.76)+(2.5 * 2.11 *$

2.2)) $=\frac{45.72}{220}=0.2$

$\operatorname{Rang}($ CT5 $)=(((I E * C 1 * C T 5)+$

$(I E * C 2 * C T 5)+(I E * C 3 * C T 5))+$

$((\mathrm{NH} * \mathrm{C4} * \mathrm{CT} \mathbf{5})+(\mathrm{NH} * \mathrm{C5} * \mathrm{CT} 5))+$

$((C * C 6 * C T 5)+(C * C 7 * C T 5)+$

$(C * C 8 * C T 5)+(S * C 9 * C T 5)+$

$(S * C 10 * C T 5)+(S * C 11 * C T 5))=$

$((0.22 * 3.6 * 0.8)+(1.4 * 0.7 * 2.2)+$

$(0.7 * 0.7 * 0.69)+(2.16 * 3 * 0.46)+$

$(2.5 * 0.56 * 0.66)+(2.16 * 2.33 *$

$0.62)+(0.4 * 1.44 * 0.31)+$

$(2.8 * 0.5 * 0.46)+(2.25 * 0.48 *$

$2.82)+(2.2 * 2.33 * 0.48)+$

$(2.5 * 2.11 * 0.5))=\frac{46.2}{220}=0.21$

AHP Ranking

\begin{tabular}{|l|l|l|}
\hline Action (City) & \% Rate & Ranking \\
\hline CT1 (Naama) & 0.21 & 2 \\
\hline CT2(Horchaia) & 0.20 & 4 \\
\hline CT3 (Ras Elma ) & 0.27 & 1 \\
\hline CT4 (Kolea) & 0.2 & 5 \\
\hline CT5 (Maghnia) & 0.21 & 3 \\
\hline
\end{tabular}

Fuzzy deduction From Pairwise

$((\boldsymbol{N H} * \boldsymbol{C 4} * \boldsymbol{C T} \mathbf{3})+(\boldsymbol{N H} * \boldsymbol{C 5} * \boldsymbol{C T} \mathbf{3})) \mathrm{F}$ Hzzy Ponderation of Criteria level one in the

$((C * C 6 * C T 3)+(C * C 7 * C T 3)+$ Naama City

$(C * C 8 * C T 3)+(S * C 9 * C T 3)+$

$(S * C 10 * C T 3)+(S * C 11 * C T 3)) \frac{\overline{\mathrm{NH}}}{1}$

$((\mathbf{0 . 2 2} * 3.6 * \mathbf{0 . 9})+(\mathbf{1 . 4} * \mathbf{0 . 7} * \mathbf{1} \mathbf{1} \mathbf{5})+$

$(0.7 * 0.7 * 1.51)+(2.16 * 3 * 1.75)+$

$(2.5 * 0.56 * 1.7)+(2.16 * 2.33$

IE : environmental impact Criteria

$0.66)+(0.4 * 1.44 * 3.6)+\left(2.8 * \mathrm{NH}^{5}\right.$. natural hazard Criteria

$1.4)+(2.25 * 0.48 * 0.44)+$

$\mathrm{C}$ : Climate Criteria

$(2.2 * 2.33 * 2.06)+(2.5 * 2.11 * \mathrm{~S}$ : Socio Economic Criteria

2.2) $)=\frac{60}{220}=0.27$

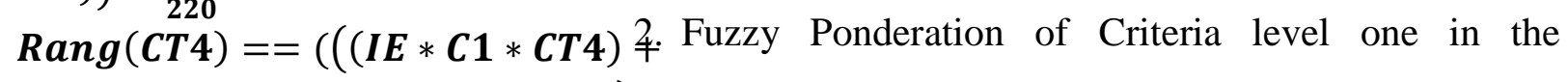

$($ IE $* C 2 * C T 4)+(I E * C 3 * C T 4))+$

$((\mathrm{NH} * \mathrm{C4} * \mathrm{CT} 4)+(\mathrm{NH} * \mathrm{C5} * \mathrm{CT} 4))+$

$((C * C 6 * C T 4)+(C * C 7 * C T 4)+$

$(C * C 8 * C T 4)+(S * C 9 * C T 4)+$

$(S * C 10 * C T 4)+(S * C 11 * C T 4))=$

$((0.22 * 3.6 * 2)+(1.4 * 0.7 * 1.3)$ Horchaia City $(\mathrm{CT} 2)$

\begin{tabular}{|l|l|l|l|l|}
\hline CT2 & IE & NH & C & S \\
\hline IE & $(1,1,1,1)$ & $(1 / 3,1 / 2,1,1)$ & $(3,4,5,6)$ & $(1 / 4,1 / 3,1 / 2,1)$ \\
\hline $\mathrm{NH}$ & $(1,2,3,4)$ & $(1,1,1,1)$ & $(6,7,8,9)$ & $(1 / 4,1 / 3,1 / 2,1)$ \\
\hline $\mathrm{C}$ & $(1 / 3,1 / 4,1 / 2,1$ & $(1 / 8,1 / 7,1 / 6,1 / 5)$ & $(1,1,1,1)$ & $(1 / 3,1 / 2,1,1)$ \\
& $(2,3,4,5)$ & $(2,3,4,5)$ & $(1,2,3,4)$ & $(1,1,1,1)$ \\
\hline $\mathrm{S}$ & $(2,3)$ & &
\end{tabular}

$(0.7 * 0.7 * 2.26)+(2.16 * 3 * 1.75) F t z z y$ Ponderation of Criteria level one in Ras ElMa

$(2.5 * 0.56 * 2.6)+(2.16 * 2.33 *$

$1.26)+(0.4 * 1.44 * 2.3)+(2.8 * 0.5 *$ 
4. City

\begin{tabular}{|l|l|l|l|l|}
\hline CT3 & IE & NH & C & S \\
\hline IE & $(1,1,1,1)$ & $(1 / 4,1 / 3,1 / 2,1)$ & $(1,1,1,1)$ & $(1 / 3,1 / 2,1,1)$ \\
\hline NH & $(2,3,4,5)$ & $(1,1,1,1)$ & $(1 / 10,1 / 9,1 / 8,1 / 7)$ & $(1 / 4,1 / 3,1 / 2,1)$ \\
\hline C & $(1,1,1,1)$ & $(8,9,10,11)$ & $(1,1,1,1)$ & $(1 / 5,1 / 4,1 / 3,1 / 2)$ \\
\hline S & $(1,2,3,4)$ & $(2,3,4,5)$ & $(3,4,5,6)$ & $(1,1,1,1)$ \\
\hline
\end{tabular}

5. Fuzzy Ponderation of Criteria level one in Maghnia City

\begin{tabular}{l|l|l|l|l|}
\hline CT4 & IE & NH & C & S \\
\hline IE & $(1,1,1,1)$ & $(1 / 10,1 / 9,1 / 8,1 / 7)$ & $(1,2,3,4)$ & $(1 / 4,1 / 3$, \\
\hline NH & $(8,9,10,11)$ & $(1,1,1,1)$ & $(1 / 6,1 / 5,1 / 4,1 / 3)$ & $(1 / 3,1 / 2,1$ \\
\hline C & $(1 / 3,1 / 2,1,1)$ & $(4,5,6,7)$ & $(1,1,1,1)$ & $(1,1,1,1)$ \\
\hline S & $(3,4,5,6)$ & $(2,3,4,5)$ & $(1,1,1,1)$ & $(1,1,1,1)$ \\
\hline
\end{tabular}
Now , The decider is invited to resolve The Sub-C
Ponderation
Ponderation of Sub-Criteria in IE
\begin{tabular}{|l|l|l|l|}
\hline IE & C1 & C 2 & $(4,5,6,7)$ \\
\hline C1 & $(1,1,1,1)$ & $(4,5,6,7)$ & $(1,1,1,1)$ \\
\hline C2 & $(1 / 6,1 / 5,1 / 4,1 / 3)$ & $(1,1,1,1)$ & $(1,1,1,1)$ \\
\hline C3 & $(1 / 6,1 / 5,1 / 4,1 / 3)$ & $(1,1,1,1)$ & \\
\hline
\end{tabular}

C1 : Pollution risk water table

$\mathrm{C} 2$ : Fauna and Flora

C3 : Citizen noise pollution
C10 : Development potential

C11 : Transport infrastructure

Then, the decider pandurate the Action as below :

Fuzzy Ponderation Of Action in C1

\begin{tabular}{|l|l|l|l|l|l|}
\hline C1 & CT1 & CT2 & CT3 & CT4 & CT5 \\
\hline CT1 & $(1,1,1,1)$ & $(1,1,1,1)$ & $(1,1,1,1)$ & $(1 / 3,1 / 2,1,1)$ & $(1,2,3,4)$ \\
\hline CT2 & $(1,1,1,1)$ & $(1,1,1,1)$ & $(1,1,1,1)$ & $(1 / 4,1 / 3,1 / 2,1)$ & $(1,1,1,1)$ \\
\hline CT3 & $(1,1,1,1)$ & $(1,1,1,1)$ & $(1,1,1,1)$ & $(1 / 3,1 / 2,1,1)$ & $(1,1,1,1)$ \\
\hline C74 & $(1,2,3,4)$ & $(2,3,4,5)$ & $(1,2,3,4)$ & $(1,1,1,1)$ & $(1,2,3,4)$ \\
\hline C55 & $(1 / 3,1 / 2,1,1)$ & $(1,1,1,1)$ & $(1,1,1,1)$ & $(1 / 3,1 / 2,1,1)$ & $(1,1,1,1)$ \\
\hline
\end{tabular}

Fuzzy Ponderation Of Action in C2 :

\begin{tabular}{|l|l|l|l|l|l|}
\hline C2 & CT1 & CT2 & CT3 & CT4 & CT5 \\
\hline C71 & $(1,1,1,1)$ & $(1 / 6,1 / 5,1 / 4,1 / 3)$ & $(1 / 4,1 / 3,1 / 2,1)$ & $(1 / 4,1 / 3,1 / 2,1)$ & $(1 / 5,1 / 4,1 / 3,1 / 2)$ \\
\hline C72 & $(4,5,6,7)$ & $(1,1,1,1)$ & $(1 / 3,1 / 2,1,1)$ & $(1,1,1,1)$ & $(1 / 3,1 / 2,1,1)$ \\
\hline CT3 & $(2,3,4,5)$ & $(1,2,3,4)$ & $(1,1,1,1)$ & $(1,1,1,1)$ & $(1 / 3,1 / 2,1,1)$ \\
\hline CT4 & $(2,3,4,5)$ & $(1,1,1,1)$ & $(1,1,1,1)$ & $(1,1,1,1)$ & $(1 / 3,1 / 2,1,1)$ \\
\hline CT5 & $(3,4,5,6)$ & $(1,2,3,4)$ & $(1,2,3,4)$ & $(1,2,3,4)$ & $(1,1,1,1)$ \\
\hline
\end{tabular}

Fuzzy Ponderation Of Action in C3

\begin{tabular}{|l|l|l|l|l|l|}
\hline C3 & CT1 & CT2 & CT3 & CT4 & CT5 \\
\hline CT1 & $(1,1,1,1)$ & $(4,5,6,7)$ & $(3,4,5,6)$ & $(2,3,4,5)$ & $(4,5,6,7)$ \\
\hline CT2 & $(1 / 6,1 / 5,1 / 4,1 / 3)$ & $(1,1,1,1)$ & $(1 / 3,1 / 2,1,1)$ & $(1 / 4,1 / 3,1 / 2,1)$ & $(1 / 3,1 / 2,1,1)$ \\
\hline CT3 & $(1 / 5,1 / 4,1 / 3,1 / 2)$ & $(1,2,3,4)$ & $(1,1,1,1)$ & $(1 / 3,1 / 2,1,1)$ & $(3,4,5,6)$ \\
\hline CT4 & $(1 / 4,1 / 3,1 / 2,1)$ & $(2,3,4,5)$ & $(1,2,3,4)$ & $(1,1,1,1)$ & $(4,5,6,7)$ \\
\hline CT5 & $(1 / 6,1 / 5,1 / 4,1 / 3)$ & $(1,2,3,4)$ & $(1 / 2,1 / 4,1 / 3,1 / 2)$ & $(1 / 6,1 / 5,1 / 4,1 / 3)$ & $(1,1,1,1)$ \\
\hline
\end{tabular}

Fuzzy Ponderation of Action in $\mathbf{C 4}$

\begin{tabular}{|c|c|c|c|c|c|c|c|}
\hline \multirow{3}{*}{\multicolumn{3}{|c|}{ Fuzzy Ponderation of Sub-Criteriatim }} & \multirow{2}{*}{ CT1CT1 } & & & & \\
\hline & & & & CT2 & CT3 & CT4 & CT5 \\
\hline & & & $\mathbf{H} \quad(1,1,1,1)$ & $(1,1,1,1)$ & $(1,1,1,1)$ & $(1,1,1,1)$ & $(2,3,4,5)$ \\
\hline $\mathrm{NH}$ & $\mathrm{C} 4$ & \begin{tabular}{l|l}
$\mathrm{C} 5$ & $\mathrm{CT} 2$ \\
\end{tabular} & $(1,1,1,1)$ & $(1,1,1,1)$ & $(1.1 .1 .1)$ & $(1,1,1,1)$ & $(2,3,4,5)$ \\
\hline $\mathrm{C} 4$ & $(1,1,1,1)$ & $(4,5,6,7)$ & & & & & \\
\hline $\mathrm{C} 5$ & $(1 / 61 / 51 / 41 / 3)$ & $\begin{array}{l}\text { CT3 } \\
\end{array}$ & $(1,1,1,1)$ & $(1,1,1,1)$ & $(1,1,1,1)$ & $(1,1,1,1)$ & $(2,3,4,5)$ \\
\hline & & CT4 & $(1,1,1,1)$ & $(1,1,1,1)$ & $(1,1,1,1)$ & $(1,1,1,1)$ & $(2,3,4,5)$ \\
\hline & & CT5 & $(1 / 4,1 / 3,1 / 2,1)$ & $(1 / 4,1 / 3,1 / 2,1)$ & $(1 / 4,1 / 3,1 / 2,1)$ & $(1 / 4,1 / 3,1 / 2,1)$ & $(1,1,1,1)$ \\
\hline
\end{tabular}

C4: Seismicity

C5 : Flood

Fuzzy Ponderation of Action in C5

Fuzzy Ponderation of sub-Criteriactn C CT1CT1

\begin{tabular}{|c|c|c|c|c|c|c|c|c|}
\hline $\mathbf{u Z z}$ & onderatio & ab-Crite & $\mathbf{a} \overline{\mathbf{p}}$ & & 12 & CT3 & CT4 & CT5 \\
\hline $\mathrm{C}$ & C6 & $\mathrm{C} 7$ & 4798 & $(1,1,1,1)$ & $(1 / 3,1 / 2,1,1)$ & $(1 / 4,1 / 3,1 / 2,1)$ & $(1 / 5,1 / 4,1 / 3,1 / 2)$ & $(1,2,3,4)$ \\
\hline C6 & $(1,1,1,1)$ & $(2,3,4,5)$ & दा(2,3,4,5 & $(1,2,3,4)$ & $(1,1,1,1)$ & $(1 / 3,1 / 2,1,1)$ & $(1 / 4,1 / 3,1 / 2,1)$ & $(1,1,1,1)$ \\
\hline $\mathrm{C7}$ & $(1 / 4,1 / 3,1 / 2,1)$ & $(1,1,1,1)$ & $f \mathbb{T}(2,3,4,5)$ & $(2,3,4,5)$ & $(1,2,3,4)$ & $(1,1,1,1)$ & $(1 / 3,1 / 2,1,1)$ & $(1,2,3,4)$ \\
\hline $\mathrm{C} 8$ & $(1 / 4,1 / 3,1 / 2,1)$ & $(1 / 4,1 / 3,1 / 2,1)$ & $\mathrm{fT}(4,1,1,1)$ & $(3,4,5,6)$ & $(2,3,4,5)$ & $(1,2,3,4)$ & $(1,1,1,1)$ & $(2,3,4,5)$ \\
\hline C6 & 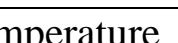 & & CT5 & $(\mathrm{1} / 3,1 / 2,1,1)$ & $(1,1,1,1)$ & $(1 / 3,1 / 2,1,1)$ & $(1 / 4,1 / 3,1 / 2,1)$ & $(1,1,1,1)$ \\
\hline
\end{tabular}

C7 : pluviometry

C8 : Bioclimatic floor

Fuzzy Ponderation of Action in C6

Fuzzy Ponderation of sub-Criteria fir $S$ CT1CT1

\begin{tabular}{|l|l|l|l|}
\hline S & C9 & C10 & C1 \\
\hline C9 & $(1,1,1,1)$ & $(1 / 4,1 / 3,1 / 2,1)$ & ( \\
\hline C10 & $(2,3,4,5)$ & $(1,1,1,1)$ & $(2,3$ \\
\hline C11 & $(4,5,6,7)$ & $(1 / 4,1 / 3,1 / 2,1)$ & $(1$, \\
\hline
\end{tabular}

C9 : Development cost

\begin{tabular}{|c|c|c|c|c|c|}
\hline $\mathrm{C} 1$ & CT1 & & $(1,1,1,1)$ & $(2,3,4,5)$ & $(1,2,3,4)$ \\
\hline$(1$ & $185,2 / 4$ & $\overline{1 / 3)}$ & $(1 / 4,1 / 3,1 / 2,1)$ & $(1,1,1,1)$ & $(2,3,4,5)$ \\
\hline$(2$, & ,4,553 & $\overline{7}$ & $1 / 3,1 / 2,1,1)$ & $(1 / 4,1 / 3,1 / 2,1)$ & $(1,1,1,1)$ \\
\hline$(1$ & $, 1,1)^{4}$ & & $(1,1,1,1)$ & $(1 / 4,1 / 3,1 / 2,1)$ & $(1,2,3,4)$ \\
\hline & CT5 & & $-(1 / 3,1 / 2,1,1)$ & $(1 / 6,1 / 5,1 / 4,1 / 3)$ & $(1,1,1,1)$ \\
\hline
\end{tabular}

\begin{tabular}{|l|l|}
\hline CT4 & CT5 \\
\hline$(1,1,1,1)$ & $(1,2,3,4)$ \\
\hline$(2,3,4,5)$ & $(4,5,6,7)$ \\
\hline$(1 / 3,1 / 2,1,1)$ & $(1,1,1,1)$ \\
\hline$(1,1,1,1)$ & $(1,2,3,4)$ \\
\hline$(1 / 3,1 / 2,1,1)$ & $(1,1,1,1)$ \\
\hline
\end{tabular}


Ponderation of Action in C7

\begin{tabular}{|c|c|c|c|c|c|}
\hline C7 & CT1 & CT2 & CT3 & CT4 & CT5 \\
\hline CT1 & $(1,1,1,1)$ & $(2,3,4,5)$ & $(1 / 4,1 / 3,1 / 2,1)$ & $(1 / 3,1 / 2,1,1)$ & $(6,7,8,9)$ \\
\hline CT2 & $(1 / 4,1 / 3,1 / 2,1)$ & $(1,1,1,1)$ & $(1 / 5,1 / 4,1 / 3,1 / 2)$ & $(1 / 3,1 / 2,1,1)$ & $(5,6,7,8)$ \\
\hline CT3 & $(2,3,4,5)$ & $(3,4,5,6)$ & $(1,1,1,1)$ & $(1,2,3,4)$ & $(7,8,9,10$ \\
\hline CT4 & $(1,2,3,4)$ & $(1,2,3,4)$ & $(1 / 3,1 / 2,1,1)$ & $(1,1,1,1)$ & $(5,6,7,8)$ \\
\hline CT5 & $(1 / 8,1 / 7,1 / 6,1 / 5)$ & $(1 / 7,1 / 6,1 / 5,1 / 4)$ & $(1 / 9,1 / 8,1 / 7,1 / 6)$ & $(1 / 7,1 / 6,1 / 5,1 / 4)$ & $(1,1,1,1)$ \\
\hline \multicolumn{6}{|c|}{ Ponderation of Action in C8 } \\
\hline $\mathrm{C} 8$ & CT1 & CT2 & CT3 & CT4 & CT5 \\
\hline CT1 & $(1,1,1,1)$ & $(1,1,1,1)$ & $(1,1,1,1)$ & $(1,1,1,1)$ & $(2,3,4,5)$ \\
\hline CT2 & $(1,1,1,1)$ & $(1,1,1,1)$ & $(1,1,1,1)$ & $(1,1,1,1)$ & $(2,3,4,5)$ \\
\hline CT3 & $(1,1,1,1)$ & $(1,1,1,1)$ & $(1,1,1,1)$ & $(1,1,1,1)$ & $(2,3,4,5)$ \\
\hline CT4 & $(1,1,1,1)$ & $(1,1,1,1)$ & $(1,1,1,1)$ & $(1,1,1,1)$ & $(2,3,4,5)$ \\
\hline CT5 & $(1 / 4,1 / 3,1 / 2,1)$ & $(1 / 4,1 / 3,1 / 2,1)$ & $(1 / 4,1 / 3,1 / 2,1)$ & $(1 / 4,1 / 3,1 / 2,1)$ & $(1,1,1,1)$ \\
\hline
\end{tabular}

\begin{tabular}{l|l|l|l|l|l|}
\multicolumn{6}{c}{ Ponderation of Action in C9 } \\
C9 & CT1 & CT2 & CT3 & CT4 & CT5 \\
\hline CT1 & $(1,1,1,1)$ & $(1 / 4,1 / 3,1 / 2,1)$ & $(1,2,3,4)$ & $(1 / 4,1 / 3,1 / 2,1)$ & $(1 / 6,1 / 5,1 / 4,1 / 3)$ \\
\hline CT2 & $(2,3,4,5)$ & $(1,1,1,1)$ & $(4,5,6,7)$ & $(2,3,4,5)$ & $(1 / 4,1 / 3,1 / 2,1)$ \\
\hline CT3 & $(1 / 3,1 / 2,1,1)$ & $(1 / 6,1 / 5,1 / 4,1 / 3)$ & $(1,1,1,1)$ & $(1 / 3,1 / 2,1,1)$ & $(1 / 6,1 / 5,1 / 4,1 / 3)$ \\
\hline CT4 & $(2,3,4,5)$ & $(2,3,4,5)$ & $(1 / 4,1 / 3,1 / 2,1)$ & $(1,1,1,1)$ & $(5,6,7,8)$ \\
\hline CT5 & $(4,5,6,7)$ & $(2,3,4,5)$ & $(4,5,6,7)$ & $(1 / 7,1 / 6,1 / 5,1 / 4)$ & $(1,1,1,1)$ \\
\hline \multicolumn{7}{|l|}{} \\
\hline C10 & CT1 & CT2 & CT3 & CT4 & CT5 \\
\hline CT1 & $(1,1,1,1)$ & $(4,5,6,7)$ & $(1,1,1,1)$ & $(1,2,3,4)$ & $(2,3,4,5)$ \\
\hline CT2 & $(1 / 6,1 / 5,1 / 4,1 / 3)$ & $(1,1,1,1)$ & $(1 / 6,1 / 5,1 / 4,1 / 3)$ & $(2,3,4,5)$ & $(1,2,3,4)$ \\
\hline CT3 & $(1,1,1,1)$ & $(4,5,6,7)$ & $(1,1,1,1)$ & $(1 / 4,1 / 3,1 / 2,1)$ & $(2,3,4,5)$ \\
\hline CT4 & $(1 / 3,1 / 2,1,1)$ & $(1 / 4,1 / 3,1 / 2,1)$ & $(2,3,4,5)$ & $(1,1,1,1)$ & $(3,4,5,6)$ \\
\hline CT5 & $(1 / 4,1 / 3,1 / 2,1)$ & $(1 / 3,1 / 2,1,1)$ & $(1 / 4,1 / 3,1 / 2,1)$ & $(1 / 3,1 / 4,1 / 5,1 / 4)$ & $(1,1,1,1)$ \\
\hline
\end{tabular}

Fuzzy Ponderation of Action in $\mathrm{C10}$

Calculate of Fuzzy Weight between different cities

Let $x(y, z)$ fonction defined as below :

$x$ : Fuzzy argument number function

$\boldsymbol{y}$ : first Intitule cities of Comparison

$y$ : Second Intitule cities of Comparison

Application

$\boldsymbol{x}(\boldsymbol{y}, \mathbf{z})=\boldsymbol{x}(\boldsymbol{y}) * \boldsymbol{x}(\mathbf{z})$

$\boldsymbol{y}(\boldsymbol{y}, \boldsymbol{z})=\boldsymbol{y}(\boldsymbol{y}) * \boldsymbol{y}(\mathrm{z})$

$\mathbf{z}(\boldsymbol{y}, \mathbf{z})=\mathbf{z}(\boldsymbol{y}) * \mathbf{z}(\mathbf{z})$

$$
\begin{aligned}
& 1(C T 2, C T 1)=0.002 \\
& 2(C T 2, C T 1)=0.02-C \operatorname{lose}(2,1) \\
& 3(C T 2, C T 1)=0.18 \\
& 4(C T 2, C T 1)=0.18 \\
& 1(C T 3, C T 1)=0.44 \\
& \{2(C T 3, C T 1)=2.25 \\
& 3(C T 3, C T 1)=4.5-\operatorname{Close}(3,1) \\
& 4(C T 3, C T 1)=20 \\
& \text { (1(CT4, CT1) }=2 \\
& 2(C T 4, C T 1)=12 \\
& 3(C T 4, C T 1)=30-C l o s e(4,1) \\
& 4(C T 4, C T 1)=120
\end{aligned}
$$

\section{Fuzzy Ponderation of Action in C11}

\begin{tabular}{|l|l|l|l|l|l|} 
C11 & CT1 & CT2 & CT3 & CT4 & CT5 \\
\hline CT1 & $(1,1,1,1)$ & $(4,5,6,7)$ & $(1,1,1,1)$ & $(1,1,1,1)$ & $(2,3,4,5)$ \\
\hline CT2 & $(1 / 6,1 / 5,1 / 4,1 / 3)$ & $(1,1,1,1)$ & $(1 / 6,1 / 5,1 / 4,1 / 3)$ & $(1 / 6,1 / 5,1 / 4), 1 / 2)$ & $(1,2,3,4)$ \\
\hline CT3 & $(1,1,1,1)$ & $(/ 4,5,6,7)$ & $(1,1,1,1)$ & $(1,1,1,1)$ & $(2,3,4,5)$ \\
\hline CT4 & $(1,1,1,1)$ & $(4,5,6,7)$ & $(1,1,1,1)$ & $(1,1,1,1)$ & $(2,3,4,5)$ \\
\hline CT5 & $(1 / 4,1 / 3,1 / 2,1)$ & $(1 / 3,1 / 2,1,1)$ & $(1 / 4,1 / 3,1 / 2,1)$ & $(1 / 4,1 / 3,1 / 2,1)$ & $(1,1,1,1)$ \\
\hline
\end{tabular}

$(1(C T 5, C T 1)=0.0002$

$2(C T 5, C T 1)=0.0004$

$3($ CT5, CT1 $)=0.066-\operatorname{Close}(5,1)$

$4($ CT5, CT1 $)=2.33$

$1(C T 1, C T 2)=2.66$

$2(C T 1, C T 2)=12.5$

$3(C T 1, C T 2)=78-\operatorname{Close}(1,2)$

$4(C T 1, C T 2)=245$

\section{Fuzzy deduction}

Rang $_{\text {Flou }}(C T i)=\left(\left(\left(I E_{F} \otimes C 1_{F} \bigotimes\right.\right.\right.$ $\left.C T i_{F}\right) \boxplus\left(I E_{F} \otimes C 2_{F} \otimes C T i_{F}\right) \boxplus$ $\left.\left(I E_{F} \otimes C 3_{F} \otimes C T i_{F}\right)\right) \boxplus\left(\left(N H_{F} \otimes C 4_{F} \otimes\right.\right.$ $\left.\left.C T i_{F}\right) \boxplus\left(N_{F} \otimes C 5_{F} \otimes C T i_{F}\right)\right) \boxplus$ $\left(\left(C_{F} \otimes C 6_{F} \otimes C T i_{F}\right) \boxplus\left(C_{F} \otimes C 7_{F} \otimes\right.\right.$ $\left.C T i_{F}\right) \boxplus\left(C_{F} \otimes C 8_{F} \otimes C T i_{F}\right) \boxplus$ $\left(S_{F} \otimes C 9_{F} \otimes C T i_{F}\right) \boxplus\left(S_{F} \otimes C 10_{F} \otimes\right.$ $\left.\left.C T i_{F}\right) \boxplus\left(S_{F} \otimes C 11_{F} \otimes C T i_{F}\right)\right)$

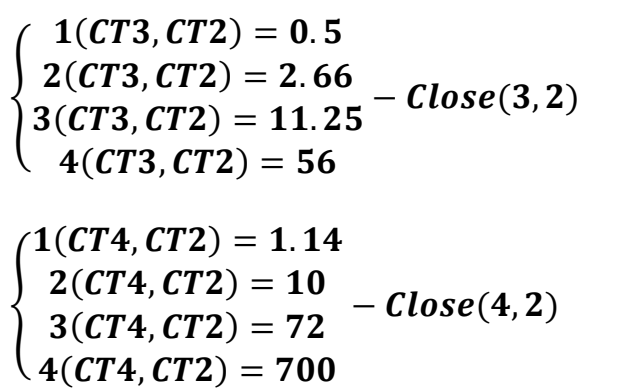




$$
\begin{aligned}
& \left\{\begin{array}{c}
1(C T 5, C T 2)=0.0003 \\
2(C T 5, C T 2)=0.003 \\
3(C T 5, C T 2)=0.01 \\
4(C T 5, C T 2)=0.4
\end{array}-\text { Close }(5,2)\right. \\
& \left\{\begin{array}{c}
1(C T 1, C T 3)=0.25 \\
2(C T 1, C T 3)=1.33 \\
3(C T 1, C T 3)=4.5 \\
4(C T 1, C T 3)=16
\end{array}\right. \\
& \left\{\begin{array}{c}
1(C T 4, C T 2)=0.01 \\
2(C T 4, C T 2)=0.07 \\
3(C T 4, C T 2)=0.5 \\
4(C T 4, C T 2)=1.9
\end{array}\right. \\
& \left\{\begin{array}{c}
1(C T 4, C T 3)=0.16 \\
2(C T 4, C T 3)=1 \\
3(C T 4, C T 3)=6 \\
4(C T 4, C T 3)=20
\end{array}-\operatorname{close}(4,3)\right. \\
& \left\{\begin{array}{c}
1(C T 5, C T 3)=0.001 \\
2(C T 5, C T 3)=0.008 \\
3(C T 5, C T 3)=0.03 \\
4(C T 5, C T 3)=1.1
\end{array}\right. \\
& \left\{\begin{array}{c}
1(C T 1, C T 4)=0.016 \\
2(C T 1, C T 4)=0.07 \\
3(C T 1, C T 4)=0.5 \\
4(C T 1, C T 4)=2
\end{array}-\operatorname{Close}(4,1)\right. \\
& \left\{\begin{array}{c}
1(C T 2, C T 4)=0.4 \\
2(C T 2, C T 4)=2.7 \\
3(C T 2, C T 4)=16 \\
4(C T 2, C T 4)=41.66
\end{array}-\operatorname{Close}(2,4)\right. \\
& \left\{\begin{array}{c}
1(C T 3, C T 4)=0.03 \\
2(C T 3, C T 4)=0.16 \\
3(C T 3, C T 4)=1.5 \\
4(C T 3, C T 4)=4
\end{array}\right. \\
& \left\{\begin{array}{c}
1(C T 5, C T 4)=0.0001 \\
2(C T 5, C T 4)=0.002 \\
3(C T 5, C T 4)=0.03 \\
4(C T 5, C T 4)=0.1
\end{array}\right. \\
& \left\{\begin{array}{c}
1(C T 1, C T 5)=8 \\
2(C T 1, C T 5)=75.6 \\
3(C T 1, C T 5)=424 \\
4(C T 1, C T 5)=1500
\end{array}-\operatorname{Close}(5,1)\right. \\
& \left\{\begin{array}{c}
1(C T 2, C T 5)=20 \\
2(C T 2, C T 5)=360 \\
3(C T 2, C T 5)=4116 \\
4(C T 2, C T 5)=28800
\end{array} \text { Close }(5,2)\right.
\end{aligned}
$$

\begin{tabular}{|c|c|c|c|c|c|c|c|}
\hline & \multicolumn{4}{|c|}{ Fuzzy Weight } & AHPw & Rank & $\%$ \\
\hline CT1 & 2.4 & 18.1 & 101.6 & 352.8 & 118.7 & 4 & $0.8 \%$ \\
\hline CT2 & 4.2 & 72.76 & 826.75 & 5769.10 & 1668.20 & 2 & $11 \%$ \\
\hline CT3 & 4.126 & 51.04 & 349.25 & 1016.2 & 355.131 & 3 & $2 \%$ \\
\hline CT4 & 120.06 & 525.4 & 9441.8 & 38568.2 & 12163.95 & 1 & $85 \%$ \\
\hline CT5 & 0.20003 & 0.2023 & 0.2027 & 1.00 & 0.26 & 5 & $0.00018 \%$ \\
\hline
\end{tabular}$$
\left\{\begin{array}{l}
1(C T 3, C T 5)=18.66 \\
2(C T 3, C T 5)=259.2 \\
3(C T 3, C T 5)=1728 \\
4(C T 3, C T 5)=5000
\end{array} \text { Close }(5,3)\right.
$$$$
\left\{\begin{array}{c}
1(C T 4, C T 5)=600 \\
2(C T 4, C T 5)=2595 \\
3(C T 4, C T 5)=47040 \\
4(C T 4, C T 5)=192000
\end{array} \text { Close }(5,4)\right.
$$$$
x\left(C T_{i}, C T_{i}\right)=(1,1,1,1)
$$

This is the diagonal Close, Its for ALL time with one We have to combine All the possibilities included in table and Calculate with function $x$ the Multiplication of

\section{Fuzzy Deduction}

\section{GIS visualization with Udig Annalyser}

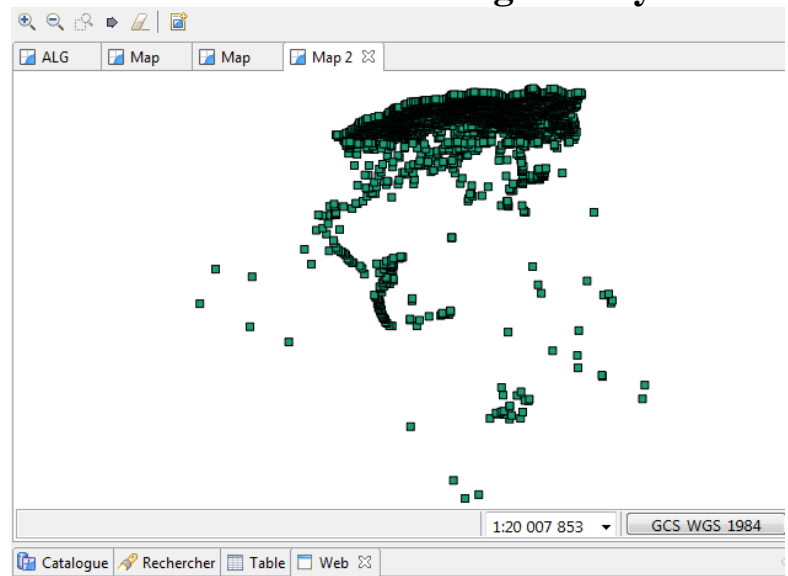

Figure 06 : Localization of Different Cities of Algeria Country

This Map have represented all Algerian Cities make on points, with Udig [18], I have characterized The latitude and longitude coordinate by rectangle function of Algerian Map, it was projected with Udig, one of famous software simulation, 


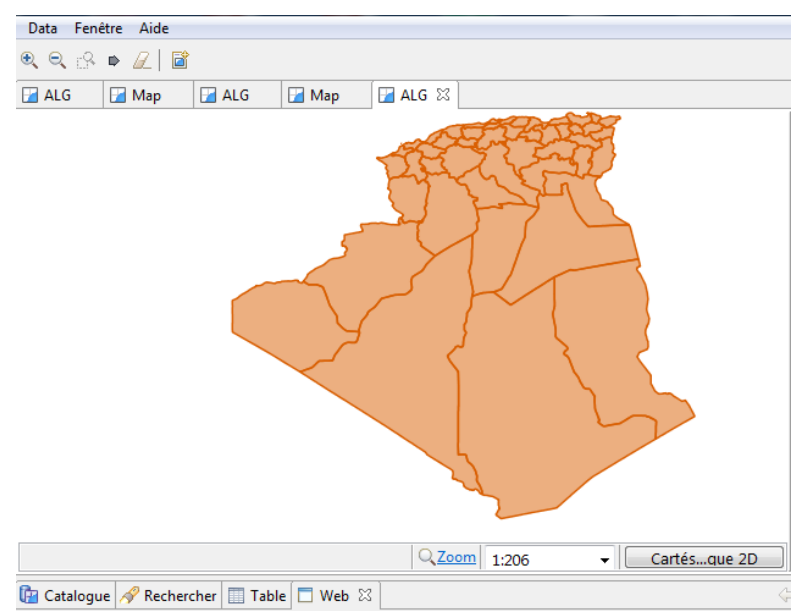

Figure 07: Administration Limitation of Algerian Cities

With Orange color, I have illustrate the Algerian Map to will determinate the borne and Limitation of each cities

\section{Conclusion}

In this paper I have proposed AHP Method then extension AHP , FAHP, and Trapezoidal model , GIS Cartography for the Algerian Industrial zone, Our Process for the decision making is based on :

1. Analysis of AHP Criteria one and second level of decision Tree

2. Analysis of AHP Alternatives on the final level in the decision Tree

3. Analysis of FAHP Criteria, the one and Second level in the decision tree

4. Analysis of FAHP of the Alternative decision in the final level on decision tree

5. Layer Visualizations of actions on GIS there are All Resource, Services and Low Favorite Climate

The final analysis had found that KOLEA (CT4) is the best Cities with Majority of Fuzzy Weight and AHP Weight to dominate The Choice Industrial Project, in another way, we Can compare the different Climate (Temperature, Climate, Socio Economic Criteria and Environmental Impact ) for extract the Rank of each one by cities, also, In Algeria , I have selected The trapezoidal Function for largeness the domain of determination

\section{REFERENCE}

[1] Chakhar, S. , "Cartographie Décisionnelle Multicritère Formalisation Et Implémentation Informatique ", Thèse de doctorat, D.F.R.
Sciences des Organisations, Université Paris Dauphine, France,2006.

[2] M.H. Vahidniaa, A. Alesheikhb, A. Alimohammadic and A. Bassirid, "A Fuzzy Analytical Hierarchy Process in GIS Application", the International Archives of the Photogrammetry, Remote Sensing and Spatial Information Sciences. Vol.37.part B2. Beijing, 2008.

[3] T. L. Saaty, "Decision making with the analytic hierarchy process", Int. J. Services Sciences, Vol. 1, No. 1, PP.83-98, 2008.

[4] Malczewski, J., "GIS-based multi-criteria decision analysis: a survey of the literature", International Journal of Geographical Information Science, Vol. 20, No. 7, PP. 703726, 2006.

[5] D.Y. Chang, "Applications of the extent analysis method on fuzzy AHP",European Journal of Operational Research, 95, PP. 649655, 1996.

[6] Taibi Aissa, Baghdad Atmani ,

"Combining Fuzzy AHP with GIS and

Decision Rules for Industrial Site Selection" PP.60-68, 2017

[7] Atmani, B., and Beldjilali, B., "Knowledge Discovery in Database: Induction Graph and Cellular Automaton", Computing and Informatics Journal, Vol.26, No. 2, PP. 171197, 2007.

[8] M.L. Vazquez, J.-P. Waaub, A. Ilinca and J. L. Chaumel, "Analyse spatiale et approche d'aide multicritères et multi acteurs a la négociation pour évaluer des scenarios d'implantation des parcs éoliens", présenté a la 1ere Conférence Intercontinentale d'Intelligence Territoriale «Interdisciplinarité dans l'aménagement et développement des territoires», PP.10, Oct. 2011, Gatineau, Canada.

[8] Dedemen, Y., "A multi-criteria decision analysis approach to GIS-based route selection for overhead power transmission lines", Thesis submitted to the Graduate School of Natural and Applied Sciences of Middle East Technical University. Department of Geodetic and Geographic Information Technologies, 2013.

[9] Y. Meng, J. Malczewski and S. Boroushaki, "A GIS-Based Multicriteria 
Decision Analysis Approach for Mapping [18] Flávio Fonseca Nobre ,Lilian Terezinha, Accessibility Patterns of Housing Ferreira Trotta, Luiz Flávio Autran Monteiro Development: A Case Study in Canmore, Gomes «Multi-criteria decision making-an Alberta", Journal of Geographic Information System, Vol. 3, PP. 50-61, 2011.

[10] S. Kubler, J. Robert, W. Derigent, A. [19] http://udig.github.io/data/

Voisin and Yves Le. Traon, "A stateof the art $\&$ testbed of fuzzy AHP (FAHP) applications", Experts Systems with Application, Vol. 65, PP. 398-422, 2016.

[11] T. Rashed and J. Weeks, "Assessing vulnerability to earthquake hazards through spatial multicriteria analysis of urban areas", International Journal of Geographical Information Sciences, Francis and Taylor edition, 2003.

[12] E. H. Ibrahim, S. E. Mohamed, and A. A. Atwan, "Combining fuzzy analytic hierarchy process and GIS to select the best location for a wastewater lift station in Elmahalla El kubra, North Egypt", International Journal of Engineering \& Technology, Vol.11, No. 05, PP. 37-43, 2011.

[13] V. Akbari, M.A. Rajabi, S.H. Chavoshi and R. Shams, "Landfill Site Selection by Combining GIS and Fuzzy Multi Criteria Decision Analysis, Case Study: Bandar Abbas, Iran", World Applied Sciences Journal, Vol. 3, PP. 39-47, 2008.

[14] S.-Y. Chou, Y.-H. Chang, C.-Y. Shen, "A fuzzy simple additive weighting system under group decision-making for facility location selection with objective/subjective attributes", European Journal of Operational Research, Vol. 189, PP.132-145, 2008.

[15] S. Sener, E. Sener, B. Nas and R. Karaguzel "Combining AHP with GIS for landfill site selection: A case study in the Lake Beys_Ehircatchment area (Konya, Turkey)", Waste Management journal, Vol. 30, 2010.

[16] S. Sener, E. Sener and R. Karagüzel, "Solid waste disposal site selection with GIS and AHP methodology: a case study in Senirkent-Uluborlu (Isparta) Basin, Turkey", Environ Monit Assess journal, 173, PP. 533 554, 2011.

[17] M. Ekmekciog ${ }^{\natural u}$, T. Kaya and C. Kahraman, "Fuzzy multicriteria disposal method and site selection for municipal solid waste", Waste Management journal Vol. 30, PP. 1729-1736, 2010.

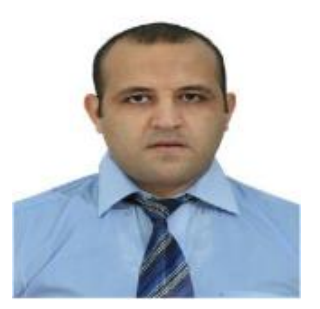

$\mathrm{He}$ is a computer science Student at the Faculty of Exact Science of Oran 1 University (Algeria). $\mathrm{He}$ earned his Master of Science degree in 2016

From Oran 1 Ahmed Ben Bella University. His research interests focus on Face, Mouth and Eyes Detection based on Classification 